\title{
Male breast carcinoma: radiotherapy contributed to favorable local control in two cases and related literature review
}

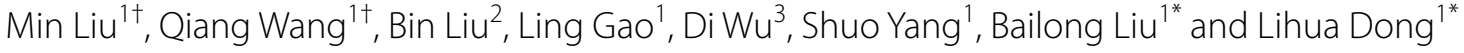

\begin{abstract}
Male breast carcinoma (MBC) is rarely encountered in clinical practice. Due to its paucity, our knowledge of MBC only rely on small or single-institutional studies and sporadic cases. The current guidelines for MBC are extrapolated from its female counterparts Rudlowski (Breast Care (Basel) 3(3):183-189, 2008). Nowadays, MBC is actively studied and viewed as a potentially different entity on the aspects of etiology, biological behavior and prognosis. Thus, special treatment strategy guidelines should be established for MBC. Additionally, advance in the systemic chemotherapy and hormonal therapy also contribute to the local control. The indication of radiotherapy need to be clarified and over-treatment should be avoided. Here we present two cases of MBC in which radiotherapy help to sustain a satisfactory disease free survival. Our cases will provide valuable experience for identifying the role of radiotherapy in MBC.
\end{abstract}

Keywords: Male breast carcinoma, Radiotherapy

\section{Background}

$\mathrm{MBC}$ is exceedingly rare, composing less than $1 \%$ of all the breast cancer [1]. Despite increasing incidence in recent years [2], many aspects such as best intervention, prognostic factors and so on are still unclear. Here, we present two rare cases of MBC. In the former, adjuvant radiotherapy contributed to prevention of local relapse. In the latter, radiotherapy was effective in controlling the recurrence of chest wall and achieved a good disease free survival (DFS) of at least 28 months.

\section{Case presentation}

\section{Case 1}

A 53-year-old man presented in January, 2011 with a history of painless lump of left breast for about 5 months. On physical examination, a hard lump measuring about $3.0 \times 2.5 \mathrm{~cm}$ was palpated in the left breast and an enlarged, irregular lymph node of $2.0 \times 1.5 \mathrm{~cm}$ was palpated in the left axilla. The breast ultrasound showed a

\footnotetext{
*Correspondence: bailong3385@163.com; lijie200461@126.com

${ }^{\dagger}$ Min Liu and Qiang Wang contributed equally to this work

${ }^{1}$ Department of Radiation Oncology, The First Hospital, Jilin University, 71

Xinmin Street, 130021 Changchun, China

Full list of author information is available at the end of the article
}

$3.33 \times 2.07 \mathrm{~cm}$ hypoechoic lesion in the left breast with blood flows and a $1.61 \times 1.2 \mathrm{~cm}$ lymph node without normal structure. BI-RADS: 4b. The following Mammotome needle biopsy of left breast lump revealed invasive ductal carcinoma (IDC) with immunohistochemical results of ER (40\%+), PR(-), Her-2(-). Relevant staging examination ruled out distant metastasis. Thus, a male breast IDC of IIB, cT2N1M0, was diagnosed.

First, the patient received neoadjuvant chemotherapy of five cycles of TAC (Docetaxel, Adriamycin and Cyclophosphamide) and attained a near complete remission response. On 4th May, 2011, modified radical mastectomy (MCM) was performed and definitive pathology showed only a little residual ductal carcinoma in situ with partial microinvasion. Left axillary dissection proved negative for nodal metastases $(0 / 29)$. The tumor bed was $1.5 \times 1.1 \times 1.0 \mathrm{~cm}$ and immunohistochemistry showed ER $(40 \%+)$, PR(-), Her-2(-). Then he received a cycle of TAC followed by adjuvant radiotherapy to left chest, supra and subclavicular region with a dose of $50 \mathrm{~Gy} / 25 \mathrm{f}$. Endocrine therapy was declined by the patient. To date, he lives a life of good quality and a 3-year DFS has been attained. 


\section{Case 2}

A 60-year-old man presented in April, 2010 with 1-month history of lump in right breast. Breast ultrasound demonstrated a $1.67 \times 1.19 \mathrm{~cm}$ hypoechoic mass in the right breast. The subsequent biopsy demonstrated breast carcinoma. Then he received MCM in another hospital. The postoperative pathology showed IDC of grade II, with component of small tube carcinoma and right axillary lymph node metastases (2/14). Immunohistochemistry showed ER(-), PR(-), Her-2(-). He was staged as IIA, pT1N1M0. Then he received 6 cycles of chemotherapy but didn't receive radiotherapy. 15 months later, recurrence of chest wall occurred and local resection was performed. The pathological results were as followings: IDC with invasion of pectoral muscle, vascular and nerve tissue, Ki-67 (+30\%), ER (+60\%), PR(-), Her-2(-). The borders were positive. The patient took tamoxifen but refused radiotherapy. 4 months later, a $2.0 \mathrm{~cm} \times 2.0 \mathrm{~cm}$ mass appeared on the chest wall near the previous recurrent site. Then radiotherapy with a dose of $70 \mathrm{~Gy} / 35 \mathrm{f}$ to the recurrence and $50 \mathrm{~Gy} / 25 \mathrm{f}$ to the chest wall was carried out and the recurrent lesion disappeared. To date, DFS has reached to 28 months.

\section{Discussion}

$\mathrm{MBC}$ is rather rare, with a predilection of elder men [3]. Despite great improvement in breast cancer treatment recently, MBC benefit less than FBC [4]. According to Surveillance, Epidemiology and End Result (SEER) data analysis, survival of breast cancer patients between 1996 and 2005 showed a $42 \%$ decrease of breast cancer-specific death in female, but only a $28 \%$ decrease in male [4, 5]. Comparing with female counterpart, MBC demonstrate more advanced stage-related tumor features such as $40 \%$ stage III or IV disease [6] and 40-55\% axillary lymph node metastasis at first visit [7] and more tumor size $>2.0 \mathrm{~cm}$ [8]. Interestingly, $\mathrm{MBC}$ manifested less advanced biology-related variables such as high tumor grade and ER or PR negativity [8]. IDC is the most common pathological type of MBC [9]. According to the multivariate analysis by Yoney et al. [9], lymph node metastasis was significantly associated with a poor DFS and overall survival (OS).

Recently, MBC is increasingly recognized as a different disease from FBC. Research have been initiated to clarify specific biomarkers of MBC pathogenesis. Genomewide microarray analysis revealed miR-10a, miR-10b, miR-125b, miR-126 and miR-191 were underexpressed in MBC samples while miR-26b, miR-135b and miR-607 were overexpressed comparing with gynecomastia samples. As a target protein of miR-126, VEGF overexpressed correspondingly, partly contributing to the angiogenesis and progression of MBC. Comparing with FBC, 4
miRNAs overexpressed and 13 underexpressed in MBC [10]. Compared to gynecomastia, prolactin receptor expression was remarkably higher in $\operatorname{MBC}[4,11]$. Survivin and $\mathrm{COX}-2$ were expressed in a substantial proportion of $\mathrm{MBC}$ individuals $[4,12]$. In the near future, more specific miRNAs and genes will be identified in involvement of MBC development and as novel therapeutic target consequently.

Due to the lack of adequate breast tissue, $\mathrm{MBC}$ tends to invade the pectoralis major muscle. MCM is recommended as the standard surgical protocol to achieve R0 resection [13]. Sentinel lymph node (SLN) analysis seemed to be reliable in MBC [8]. In early-staged patients, breast conserving surgery might be feasible [4, 14].

In the past, radiotherapy was performed for all the postoperative $\mathrm{MBC}$ patients in view of inadequate surgical margin due to the lack of breast tissue $[13,15]$. Yu et al. also indicated that postmastectomy radiotherapy (PMRT) bring significantly better local relapse free survival rather than OS benefit, especially for individuals with high risk factors such as $\leq 2 \mathrm{~mm}$ or unknown surgical margin, advanced stage and lymph node metastasis [16]. Now, PMRT indication in MBC follow recommendations for $\mathrm{FBC}$, especially for those with axillary nodal involvement [17]. Besides, more $\mathrm{MBC}$ were inclined to PMRT than FBC for higher possibility of skin and nipple involvement $[18,19]$. Korde et al. considered that retroareolar tumor or muscle invasion were also PMRT indication in MBC [8]. These two cases should be subjected to PMRT due to the involvement of axillary lymph nodes. The timely radiotherapy after MCM contributed to long-term DFS in the first patient. However, in the second case who didn't receive PMRT, chest wall recurrence took place 15 months after surgery. The first 2 years was the peak of recurrence [9]. Radiotherapy after recurrence also played a critical role in local control. According to a 20-year survival data for PMRT in MBC, stage III disease could obtain OS benefit from PMRT whereas stages I and II could not. However, the results of this retrospective research was questioned for adverse long-term effects in earlier stages caused by obsolete irradiation techniques impaired the OS benefits [20]. Advanced radiotherapy techniques nowadays will maximize the dose to target volume and minimize the dose of normal organs, which might transform to the OS benefit for early-staged MBC patients.

$90 \% \mathrm{MBC}$ cases express hormonal receptor. Thus, hormonal treatment is a crucial part of the management strategy [21]. Tamoxifen contributes to improved DFS and OS [22]. The application of aromatase inhibitors in $\mathrm{MBC}$ is still worthy of scrutiny [2]. The main reason is that testicular production of estrogen is independent 
of aromatase, composing about $20 \%$ circulating estrogen $[2,23]$. Furthermore, aromatase inhibitors has been reported to induce the increase of testosterone level providing more substrate for the production of estrogen $[2,24]$. However, there is a high rate of discontinuation of tamoxifen in MBC due to one or more toxicity. Most common toxic effects are sexual dysfunction and weight gain [25]. In the second case, Tamoxifen alone failed to prevent the chest wall relapse while radiotherapy exerted favorable effect in local control. Because most of MBC patients are ER positive and older, adjuvant chemotherapy can benefit $\mathrm{MBC}$ with high risks such as young age, endocrine-nonresponse, high tumor grade and multiple axillary lymph node involvement [8].

Because of its rarity, only limited reports of small samples or single institute can be reviewed [26]. Cutuli et al. analyzed $489 \mathrm{MBC}$ cases in their institute and revealed the similar outcomes to FBC after early diagnosis and wide application of adjuvant treatments such as radiotherapy, hormonal and chemotherapy [27]. However, biological differences between $\mathrm{MBC}$ and FBC should be emphasized and the treatment guidelines should not simply extrapolate FBC algorithms. In the future, collaboration of multiple institutions should be initiated and longer follow-up are fundamental for the full-scale research of this entity [26].

\section{Conclusion}

MBC is now recognized as an entity with a potentially different biology and special treatment algorithms for MBC should be established in the near future. Radiotherapy indication for MBC patients need to be identified. Collaborative clinical trials are critical to clarify the optimal treatment for MBC.

\section{Consent}

Written informed consent was obtained from the patient for publication of this case report and accompanying images. A copy of the written consent is available for review by the Editor-in-Chief of this journal.

\section{Abbreviations \\ DFS: disease free survival; ER: estrogen receptor; FBC: female breast carcinoma; IDC: invasive ductal carcinoma; MBC: male breast carcinoma; MCM: modified radical mastectomy; OS: overall survival; PMRT: postmastectomy radiotherapy; PR: progesterone receptor; SEER: surveillance, epidemiology and end result; SLN: sentinel lymph node.}

\section{Authors' contributions}

$M L$ was a major contributor in writing the manuscript. QW, BL and BL revised it critically. DW, $L G$ and $S Y$ reviewed $M B C$ treatment. $B L$ and $L D$ reviewed the literature and gave final approval of the version to be submitted. All authors read and approved the final manuscript.

\section{Author details}

'Department of Radiation Oncology, The First Hospital, Jilin University, 71 Xinmin Street, 130021 Changchun, China. ${ }^{2}$ Department of Hand Surgery, The First Hospital, Jilin University, 71 Xinmin Street, 130021 Changchun, China.

${ }^{3}$ Department of Breast Surgery, The First Hospital, Jilin University, 71 Xinmin Street, 130021 Changchun, China.

\section{Acknowledgements}

The present study was supported by Science and Technology Department of Jilin Province (Grant no: 3D512J233428), the Natural Science Foundation of China (Grant no: 81272999), the First Hospital of Jilin University (Grant no: JDYY52015004, JDYY52015012) and Traditional Chinese Medicine Administration Bureau of Jilin Province (Grant no: 2014-Q54).

\section{Competing interests}

The authors declare that they have no competing interests.

Received: 5 December 2014 Accepted: 9 September 2015

Published online: 26 November 2015

\section{References}

1. Jemal A, Thomas A, Murray T, Thun M. Cancer statistics 2002. CA Cancer J Clin. 2002;52(1):23-47.

2. Nahleh ZA. Hormonal therapy for male breast cancer: a different approach for a different disease. Cancer Treat Rev. 2006;32(2):101-5.

3. Hultborn R, Friberg S, Hultborn KA, Peterson LE, Ragnhult I. Male breast carcinoma. II. A study of the total material reported to the Swedish cancer Registry $1958 \pm 1967$ with respect to treatment, prognostic factors and survival. Acta Oncol. 1987:26(5):327-41.

4. Onami S, Ozaki M, Mortimer JE, Pal SK. Male breast cancer: an update in diagnosis, treatment and molecular profiling. Maturitas. 2010;65(4):308-14.

5. Anderson WF, Jatoi I, Tse J, Rosenberg PS. Male breast cancer: a population-based comparison with female breast cancer. J Clin Oncol. 2010;28(2):232-9.

6. Rudlowski C. Male breast cancer. Breast Care (Basel). 2008;3(3):183-9

7. Borgen PI, Wong GY, Vlamis V, Potter C, Hoffmann B, Kinne DW, Osborne MP, McKinnon WM. Current management of male breast cancer. A review of 104 cases. Ann Surg. 1992;215(5):451-7.

8. Korde LA, Zujewski JA, Kamin L, Giordano S, Domchek S, Anderson WF, Bartlett JM, Gelmon K, Nahleh Z, Bergh J, Cutuli B, Pruneri G, McCaskillStevens W, Gralow J, Hortobagyi G, Cardoso F. Multidisciplinary meeting on male breast cancer: summary and research recommendations. J Clin Oncol. 2010:28(12):2114-22.

9. Yoney A, Kucuk A, Unsal M. Male breast cancer: a retrospective analysis. Cancer Radiother. 2009;13(2):103-7.

10. Fassan M, Baffa R, Palazzo JP, Lloyd J, Crosariol M, Liu CG, Volinia S, Alder H, Rugge M, Croce CM, Rosenberg A. MicroRNA expression profiling of male breast cancer. Breast Cancer Res. 2009;11(4):R58.

11. Ferreira M, Mesquita $M$, Quaresma $M$, André S. Prolactin receptor expression in gynaecomastia and male breast carcinoma. Histopathology. 2008;53(1):56-61

12. Younis T, Haché KD, Rayson D, Dewar R, Gray S, Barnes PJ. Survivin and COX-2 expression in male breast carcinoma. Breast. 2009:18(4):228-32.

13. Stranzl H, Mayer R, Quehenberger F, Prettenhofer U, Willfurth P, Stöger H, Hackl A. Adjuvant radiotherapy in male breast cancer. Radiother Oncol. 1999;53(1):29-35

14. Golshan M, Rusby J, Dominguez F, Smith BL. Breast conservation for male breast carcinoma. Breast. 2007;16(6):653-6.

15. Robison R, Montague ED. Treatment results in males with breast cancer. Cancer. 1982;49(2):403-6.

16. Yu E, Suzuki H, Younus J, Elfiki T, Stitt L, Yau G, Vujovic O, Perera F, Lock $\mathrm{M}$, Tai $\mathrm{P}$. The impact of post-mastectomy radiation therapy on male breast cancer patients: a case series. Int J Radiat Oncol Biol Phys. 2012:82(2):696-700 
17. Cutuli B. Strategies in treating male breast cancer. Expert Opin Pharmacother. 2007;8(2):193-202.

18. Scott-Conner CE, Jochimsen PR, Menck HR, Winchester DJ. An analysis of male and female breast cancer treatment and survival among demographically identical pairs of patients. Surgery. 1999;126(4):775-80 (discussion 780-771)

19. Atahan L, Yildiz F, Selek U, Sari S, Gurkaynak M. Postoperative radiotherapy in the treatment of male breast carcinoma: a single institute experience. J Natl Med Assoc. 2006;98(4):559-63.

20. Eggemann H, Ignatov A, Stabenow R, von Minckwitz G, Röhl FW, Hass P, Costa SD. Male breast cancer: 20-year survival data for post-mastectomy radiotherapy. Breast Care (Basel). 2013;8(4):270-5.

21. Fentiman IS, Fourquet A, Hortobagyi GN. Male breast cancer. Lancet. 2006;367(9510):595-604.

22. Goss PE, Reid C, Pintilie M, Lim R, Miller N. Male breast carcinoma: a review of 229 patients who presented to the Princess Margaret Hospital during 40 years: 1955-1996. Cancer. 1999;85(3):629-39.
23. Handesman D. Androgen actions and pharmacologic uses. In: Detroit IJ, editor. Endocrinology. Philadelphia: WB Saunders; 2001. p. 2232-42.

24. Harris AL, Dowsett M, Stuart-Harris R, Smith IE. Role of aminoglutethimide in male breast cancer. Br J Cancer. 1986;54(4):657-60.

25. Pemmaraju N, Munsell MF, Hortobagyi GN, Giordano SH. Retrospective review of male breast cancer patients: analysis of tamoxifenrelated side-effects. Ann Oncol. 2012;23(6):1471-4.

26. Sedighi A, Hamed EA, Mohammadian K, Behnood S, Kalaghchi B. Clinicopathologic characteristics of male breast cancer: a report of 21 cases in radiotherapy center of Hamedan, Iran. Asian Pac J Cancer Prev. 2013;14(12):7381-3.

27. Cutuli B, Le-Nir CC, Serin D, Kirova Y, Gaci Z, Lemanski C, De Lafontan B, Zoubir M, Maingon P, Mignotte H, de Lara CT, Edeline J, Penault-Llorca F, Romestaing P, Delva C, Comet B, Belkacemi Y. Male breast cancer. Evolution of treatment and prognostic factors. Analysis of 489 cases. Crit Rev Oncol Hematol. 2010;73(3):246-54.

\section{Submit your next manuscript to BioMed Central and take full advantage of:}

- Convenient online submission

- Thorough peer review

- No space constraints or color figure charges

- Immediate publication on acceptance

- Inclusion in PubMed, CAS, Scopus and Google Scholar

- Research which is freely available for redistribution

Submit your manuscript at www.biomedcentral.com/submit 